\title{
Red Blood Cell Mechanical Stability
}

\author{
Oguz K. Baskurt \\ Koc University School of Medicine, Rumelifeneri Kampusu, Sariyer, Istanbul, Turkey \\ Email: obaskurt@ku.edu.tr \\ Received 2012
}

\begin{abstract}
It has been well documented that shear forces effective above a certain magnitude under flow conditions causes damage in blood cells. This damage ranges from slight morphological alteration to the destruction of red blood cells (RBC). The hemolytic trauma to RBC can easily be detected by measuring free hemoglobin level in plasma, however there are no standardized protocols to quantitate subhemolytic trauma. Ektacytometry has been used to study the alterations in RBC mechanical properties induced by the application of shear stress at various levels. Additionally, a protocol for measuring the hemolytic threshold as an indicator of subhemolytic damage to RBC has been developed based on ektacytometry. These standardized protocols may find applications in the pre-clinical and clinical evaluation of artificial organs and biomedical devices contacting with blood.
\end{abstract}

Keywords: Artificial Organs; Blood Damage; Subhemolytic Damage; Ektacytometry

\section{Introduction}

Red blood cells (RBC) are exposed to mechanical stresses during their "everyday life", at various levels of the circulatory system. They respond to this effect by changing their shape and orientation to the flow conditions; but they are not overtly damaged by these forces, under physiological conditions. However, if they are exposed to non-physiological conditions, like in case of circulating through artificial organs or devices, they might be affected resulting in deteriorations at various degrees, ranging from slight morphological alterations to the rupture of their membrane resulting in hemolysis [9].

The most effective factors under such conditions include the extremely high shear forces to which RBC are exposed to during their passage through the artificial flow environments [5, 9]. Additionally, turbulence, cavitation, contact of blood cells with artificial surfaces, and collision between cells or betweens cells and various parts of the device may also damage the cellular components of blood [5, 9]. Shear forces in the human arterial system are in the range of 1-10 Pascal (Pa) [7], while in artificial circulatory environments (e.g., ventricular assist devices, total artificial heart, extracorporeal circulation systems, heart valves, stents, hemodialysis systems) these forces may approach to several hundred $\mathrm{Pa}$ [5].

\section{Mechanical Trauma to Blood}

It is well known for many decades that shear forces exceeding a critical level may cause hemolysis [12]. It has been demonstrated that both magnitude and exposure time are important determinants of the hemolytic effect of shear stress [6]. Hemolytic damage to RBC can easily be quantitated by measuring free hemoglobin in the suspending medium of blood (plasma), which is usually expressed as the ratio to the total hemoglobin content of whole blood. This hemolysis ratio or its normalized forms are widely used in expressing the extent of mechanical trauma to blood and used for the evaluation of artificial circu- latory environments [9].

However, it should be noted that hemolysis is the end-stage damage to RBC, corresponding to the destruction of the cell. It has been documented that the mechanical impact to RBC under the hemolytic threshold also induce alterations in the cellular properties, at varying degrees determined by the magnitude of the effective forces $[9,15]$. It has been argued that even physiological shear forces under normal circulatory conditions induce slight changes in RBC properties, which may accumulate during the life-span of RBC ( 120 days for human RBC). This "non-lethal" trauma to RBC might be enhanced if they are exposed to the conditions in artificial circulatory environment [9].

Subhemolytic trauma might be manifest by shortened life span [3], increased trapping in spleen [14], morphological alterations [13], or deterioration in mechanical properties of the cells [2]. Subhemolytic trauma is also determined by both the magnitude and duration of the effective shear stresses [2,9].

RBC membrane stability is affected by subhemolytic trauma, resulting in increased mechanical fragility indicated by higher rate of hemolysis during the subsequent exposure to extreme shear forces. Various tests to determine mechanical fragility has been developed and proposed to be used in testing the artificial circulatory environments [4,8]. Mechanical fragility is expressed as percent free hemoglobin following a standardized mechanical stress to RBC suspensions [9]. However, the magnitude of the mechanical impact to which RBC are exposed cannot be standardized or even accurately estimated with the proposed protocols [4,8].

\section{Detection of Subhemolytic Damage to Red Blood Cells}

Accurate quantification of subhemolytic damage to RBC is important in studies aiming to evaluate the blood damage induce by artificial organs and biomedical devices contacting with blood. Additionally, test protocols and models based on subhemolytic levels of mechanical trauma to RBC may help 
designers to improve the safety limits of the artificial circulatory systems, with respect to blood trauma.

It has been demonstrated that a Couette-type viscometer is a useful apparatus to apply shear stresses with well-defined and constant geometry in a wide range of magnitude. Instruments equipped with a laser-diffraction analysis capability in addition to a shearing apparatus have been used to analyze the alterations in the deformability of RBC exposed to subhemolytic levels of shear stress [2,10,11]. Application of $120 \mathrm{~Pa}$ of shear stress for periods of 15 to 120 seconds to RBC suspensions resulted in a deterioration in deformability, the degree of impairment in elongation indexes being a function of the duration of the mechanical stress [2]. Figure 1 demonstrates the shear stress-elongation index curves of RBC suspensions before and after the application of $100 \mathrm{~Pa}$ shear stress for $300 \mathrm{~s}$. The impairment of deformability under a standardized shearing protocol has been observed to reflect the "health" of RBC prior to the application of the shear stress; a subhemolytic trauma to $\mathrm{RBC}$ (e.g., due to circulating in a artificial organ) relates to a higher level of impairment following the shearing. RBC with altered membrane stability (e.g., due to exposure to lanthanides of various ionic radius) were characterized by different degrees of impairment in deformability following a standard application of shear stress (i.e., $290 \mathrm{~Pa}$ for $300 \mathrm{~s}$ ) [1]. These observations indicate that ektacytometry can be used as a standardized, subhemolytic mechanical damage test.

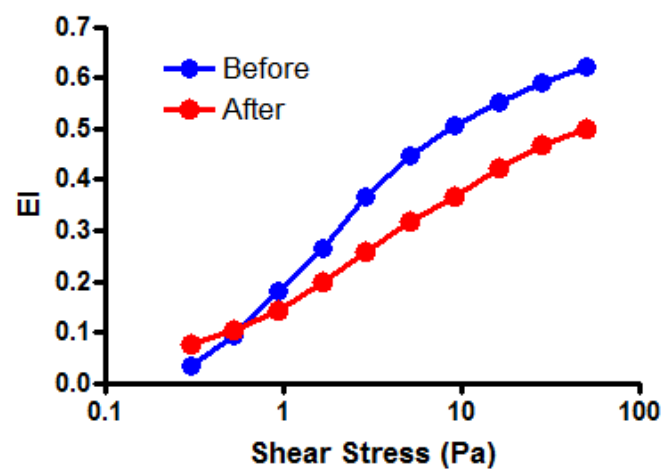

Figure 1. Shear stress-elongation index curves for red blood cell suspensions before and after being exposed to 100 Pascal shear stress for 300 seconds.

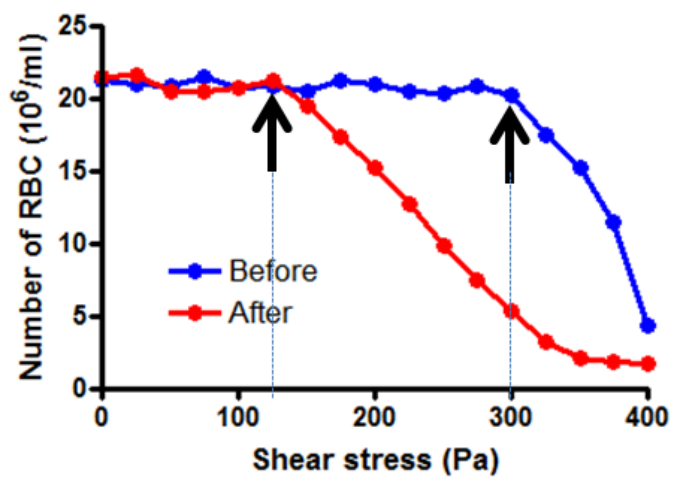

Figure 2. Number of red blood cells (RBC) in suspensions exposed to gradually increasing shear stress before and after the application of $\mathbf{1 0 0}$ Pascal shear stress for $\mathbf{3 0 0}$ seconds. The number of RBC was estimated by the area of elliptical diffraction pattern.

\section{Hemolytic Threshold Determined by Ektacytometry}

Normal RBC can withstand to shear stresses up to $300 \mathrm{~Pa}$, without being hemolysed. However, alterations in membrane stability may significantly reduce this hemolytic threshold. It is worthwhile to measure this hemolytic threshold as an indicator of the accumulated subhemolytic damage to RBC during previous life-span (e.g., in an artificial organ system).

Ektacytometry might be used as an easily applicable test protocol to measure the hemolytic threshold, as an indicator of subhemolytic trauma to RBC. The coaxial-cylindrical shearing apparatus of an ektacytometer has been used to apply gradually increasing shear stresses (in the range of 0 to $400 \mathrm{~Pa}$ ) to $\mathrm{RBC}$ suspensions and the laser diffraction images have been monitored. It has been recognized that elongation indexes remained relatively constant while the area of the elliptical diffraction pattern has changed more significantly during the course of shear stress application. Preliminary data indicates that the ellipse area is an accurate indicator of the number of RBC in the suspension under test. Figure 2 demonstrates the alterations in the number of RBC as a function of gradually increasing shear stress. The curve labeled as "Before" reflects the change in the number of RBC in suspensions before being exposed to shear stress, which indicates a sharp decrease in RBC number as shear stress approaches to $300 \mathrm{~Pa}$. This level of shear stress is accepted as the hemolytic threshold. The curve labeled as "After" reflects the same test applied to RBC suspensions following a prior exposure to $100 \mathrm{~Pa}$ shear stress for $300 \mathrm{~s}$, indicating a very significantly reduced hemolytic threshold.

\section{Conclusion}

Ektacytometry can be developed into a useful approach in studying the subhemolytic mechanical trauma to blood, to be used in the pre-clinical and clinical evaluation of artificial organs and biomedical devices contacting with blood.

\section{REFERENCES}

[1] T. Alexy, O.K. Baskurt, N. Nemeth, M. Uyuklu, R.B. Wenby and H.J. Meiselman, Effect of lanthanides on red blood cell deformability and response to mechanical stress: role of lanthanide ionic radius, Biorheology 48 (2011), 173-183.

[2] O.K. Baskurt, M. Uyuklu and H.J. Meiselman, Protection of erythrocytes from sub-hemolytic mechanical damage by nitric oxide mediated inhibition of potassium leakage, Biorheology 41 (2004), 79-89.

[3] D.E. Brinsfield, M.A. Hoff, R.B. Geering and P.M. Galletti, Hematological changes in long term perfusion, J Appl Physiol 17 (1962), 531-538.

[4] R.G. Cooper, R.A. Kahn, C.N. Cornell and M.E. Muhrer, Erythrocyte mechanical fragility test, J Clin Pathol 21 (1968), 781-783.

[5] S. Deutsch, J.M. Tarbell, K.B. Manning, G. Rosenberg and A.A Fontaine, Experimental fluid mechanics of pulsatile artificial blood pumps, Ann Rev Fluid Mech 38 (2006), 65-86.

[6] M. Giersiepen, L.J. Wurzinger, R. Opitz and H. Reul, Estimation of shear stress-related blood damage in heart valve prostheses- in vitro comparison of 25 aortic valve, Artif Organs 13 (1990), 300-306. 
[7] J.M. Greve, A.S. Les, B.T. Tang, M.T. Draney Blomme, N.M. Wilson, R.L. Dalman, N.J. Pelc and C.A. Taylor, Allometric scaling of wall shear stress from mice to humans: quantification using cine phase-contrast MRI and computational fluid dynamics, Am J Physiol Heart Circ Physiol 291 (2006), H1700-H1708.

[8] L. Gu, W.A. Smith and G.P. Chatzimavroudis, Mechanical fragility calibration of red blood cells, Asaio J 51 (2005), 194-201.

[9] M.V. Kameneva and J.F. Antaki, Mechanical trauma to blood, in: O.K.Baskurt, M.R.Hardeman, M.W.Rampling and H.J.Meiselman (Eds.), Handbook of Hemorheology and Hemodynamics, Amsterdam, Berlin, Oxford, Tokyo, Washington DC, 2007, pp. 206-227.

[10] S.S. Lee, K.H. Ahn, S.J. Lee, K. Sun, P.T. Goedhart and M.R. Hardeman, Shear induced damage of red blood cells monitored by the decrease of their deformability, Korea-Australia Rheology Journal 16 (2004), 141-146.
[11] P.J. Marascalco, S.P. Ritchie, T.A. Snyder and M.V. Kameneva, Development of standard tests to examine viscoelastic properties of blood of experimental animals for pediatric mechanical support device evaluation, Asaio J 52 (2006), 567-574.

[12] C.G. Nevaril, E.C. Lynch, C.P. frey Jr and J.D. Hellums, Erythrocyte damage and destruction induced by shear stress, $J$ Lab Clin Med 71 (1968), 784-790.

[13] E.A. Orear, M.M. Udden, J.A. Farmer, L.V. McIntire and E.C. Lynch, Increased Intracellular Calcium and Decreased Deformability of Erythrocytes from Prosthetic Heart-Valve Patients, Clinical Hemorheology 4 (1984), 461-471.

[14] J.G. Sandza, R.E. Clark, C.S. Weldon and S.P. Sutera, Subhemolytic trauma of erythrocytes; recognition and sequestration by spleen as a function of shear, ASAIO Trans 2 (1974), 457-462.

[15] S.P. Sutera, Flow-induced trauma to blood cells, Circ Res 41 (1977), 2-8 